\title{
Secretory leukocyte protease inhibitor gene deletion alters bleomycin-induced lung injury, but not development of pulmonary fibrosis
}

\author{
Anthony N Habgood', Amanda L Tatler', Joanne Porte', Sharon M Wahl', Geoffrey J Laurent ${ }^{3}$, Alison E John', \\ Simon R Johnson ${ }^{1}$ and Gisli Jenkins ${ }^{1}$
}

Idiopathic pulmonary fibrosis is a progressive, fatal disease with limited treatment options. Protease-mediated transforming growth factor- $\beta$ (TGF- $\beta$ ) activation has been proposed as a pathogenic mechanism of lung fibrosis. Protease activity in the lung is tightly regulated by protease inhibitors, particularly secretory leukocyte protease inhibitor (SLPI). The bleomycin model of lung fibrosis was used to determine the effect of increased protease activity in the lungs of Slpi ${ }^{-1-}$ mice following injury. Slpi ${ }^{-1-}$, and wild-type, mice received oropharyngeal administration of bleomycin (30 IU) and the development of pulmonary fibrosis was assessed. Pro and active forms of matrix metalloproteinase (MMP)-2 and MMP-9 were measured. Lung fibrosis was determined by collagen subtype-specific gene expression, hydroxyproline concentration, and histological assessment. Alveolar TGF- $\beta$ activation was measured using bronchoalveolar lavage cell pSmad 2 levels and global TGF- $\beta$ activity was assessed by pSmad2 immunohistochemistry. The active-MMP-9 to proMMP-9 ratio was significantly increased in S/pi ${ }^{-1-}$ animals compared with wild-type animals, demonstrating enhanced metalloproteinase activity. Wild-type animals showed an increase in TGF- $\beta$ activation following bleomycin, with a progressive and sustained increase in collagen type I, alpha 1 (Col1a1), III, alpha 1(Col3a1), IV, alpha 1(Col4a1) mRNA expression, and a significant increase in total lung collagen 28 days post bleomycin. In contrast Slpi ${ }^{-1-}$ mice showed no significant increase of alveolar TGF- $\beta$ activity following bleomycin, above their already elevated levels, although global TGF- $\beta$ activity did increase. Slpi ${ }^{-1-}$ mice had impaired collagen gene expression but animals demonstrated minimal reduction in lung fibrosis compared with wild-type animals. These data suggest that enhanced proteolysis does not further enhance TGF- $\beta$ activation, and inhibits sustained Col1a1, Col3a1, and Col4a1 gene expression following lung injury. However, these changes do not prevent the development of lung fibrosis. Overall, these data suggest that the absence of Slpi does not markedly modify the development of lung fibrosis following bleomycin-induced lung injury.

Laboratory Investigation (2016) 96, 623-631; doi:10.1038/labinvest.2016.40; published online 14 March 2016

Idiopathic pulmonary fibrosis (IPF) is a fibroproliferative disorder that leads to architectural remodeling of the lung with detrimental effects on pulmonary function. It is a chronic, progressive disease with limited therapeutic options and the prognosis remains very poor with a median survival of $\sim 3$ years. ${ }^{1}$ The etiology and underlying pathophysiology of IPF remain unclear. The current paradigm suggests that injury to the alveolar epithelium leads to an exaggerated or dysregulated fibrotic response. ${ }^{2,3}$ Although the role of inflammation in the pathogenesis of IPF remains controversial, neutrophilic polymorphonuclear leukocytes (neutrophils) have been associated with severe, progressive fibrosis. ${ }^{4}$
Neutrophil granules contain serine proteases such as neutrophil elastase (NE), cathepsin G, and proteinase-3. These granular contents are released in large quantities from activated neutrophils ${ }^{5}$ and their substrates include most matrix proteins, particularly elastin, and, to a lesser extent, collagen, laminin, fibronectin, and vitronectin. ${ }^{6}$ Neutrophil proteases have been implicated in pulmonary fibrosis. Both $\mathrm{Ne}^{-1-}$ mice and animals treated with inhibitors of NE are resistant to bleomycin-induced pulmonary fibrosis. ${ }^{7-9}$ Furthermore, levels of NE are increased in both bronchoalveolar lavage fluid and the lung parenchyma ${ }^{10,11}$ from

\footnotetext{
${ }^{1}$ Nottingham Respiratory Research Unit, University of Nottingham, Nottingham, England; ${ }^{2}$ National Institute of Dental \& Craniofacial Research, NIH, Bethesda, MA, USA and ${ }^{3}$ Lung Institute of Western Australia, The University of Western Australia, Perth, Australia

Correspondence: Mr A Habgood, BSc, Nottingham Respiratory Research Unit, University of Nottingham, Nottingham University Hospitals, Clinical Sciences Building, City Hospital Campus, Nottingham NG5 1PB, UK.

E-mail: anthony.habgood@nottingham.ac.uk

Received 15 September 2015; revised 22 January 2016; accepted 5 February 2016
} 
patients with IPF. The mechanism through which proteases promote fibrosis remains unclear, although serine proteases have been shown to activate transforming growth factor- $\beta$ (TGF- $\beta$ ) directly via proteolytic cleavage of the latency associated peptide in vitro ${ }^{12-14}$ and indirectly through activation of $\alpha \mathrm{v}$-containing integrins. ${ }^{15,16}$

Protease activity in the lung is regulated by a number of protease inhibitors. Secretory leukocyte protease inhibitor (SLPI) is an $11.7 \mathrm{kDa}$ protein containing a protease inhibitory site located at leucine 72 in the carboxy-terminal domain. ${ }^{17}$ SLPI is predominately secreted by epithelial cells ${ }^{18}$ and in the absence of SLPI, elastolytic activity in dermal and mucosal wounds has been shown to be enhanced resulting in enhanced active TGF- $\beta$, prolonged wound repair and increased scarring, ${ }^{19,20}$ suggesting that proteases may activate TGF- $\beta$ and promote fibrosis. ${ }^{19}$

This study aims to determine the role of SLPI-regulated protease activation on the development of pulmonary fibrosis. Wild-type mice had an increase in collagen gene expression, total lung collagen and phosphorylation of Smad2 28 days following bleomycin instillation, whereas $S l p i^{-1-}$ mice had evidence of increased metalloproteinase activity and more severe acute injury. However, increases in collagen gene expression in Slpi ${ }^{-1-}$ mice were not sustained over 28 days and animals demonstrated minimal reduction in lung fibrosis compared with wild-type animals. These data suggest that enhanced proteolysis in $S l \mathrm{pi}^{i^{-1}}$ mice may promote acute lung injury; however, this ultimately has little effect on the severity of fibrosis when compared with wild-type animals.

\section{MATERIALS AND METHODS \\ Animals}

Slpi $i^{-1-}$ mice on a B6D2F1 background were kindly donated by Sharon Wahl. ${ }^{19}$ This recombinant strain is now available at The Jackson Laboratory Repository with JAX Stock No. 010926 (http://jaxmice.jax.org/query). C57Bl6/j mice were obtained from Charles River UK (Margate, Kent, UK). Slpi $i^{-1-}$ mice were backcrossed by mating with C57Bl6/j for at least five generations within the Biomedical Services Unit, University of Nottingham. Animals had free access to food (Tekland Global 18\% protein rodent diet; Bicester, Oxen, UK) and water. Ear notch biopsies from animals were analyzed by polymerase chain reaction (PCR). The presence of the Slpi allele was detected by primers: sense- 5 '-CAAGGC CTTCTGTGTGTAACTTTC- $3^{\prime}$ and antisense- $5^{\prime}$-CTGCTA CAGAGTAGGTGGCAGAC-3', resulting in a 490 -bp PCR product and the Neo-cassette allele by primers: sense- $-5^{\prime}$-CG CTTCCTCGTGCTTTACGGTATC- $3^{\prime}$ and antisense- $5^{\prime}$-GA TGCCAGGCATTTGCACTGCCG -3', resulting in a 344 -bp product.

\section{Bleomycin Model}

All animal care and procedures were approved by the University of Nottingham Ethical Review Committee and were performed under Home Office Project and Personal
License authority within the Animal (Scientific Procedures) Act 1986. Mice, 6-8 weeks old, were anaesthetized with isoflurane-anesthetic and exposed to $30 \mathrm{IU}$ of bleomycin sulfate (Kyowa Hakko, Slough, UK) in $50 \mu \mathrm{l}$ sterile $0.9 \%$ saline (Sigma Aldrich), or $50 \mu \mathrm{l}$ sterile $0.9 \%$ saline control, via oropharyngeal administration.

\section{Histology}

Twenty-eight days post bleomycin the trachea was cannulated and the pulmonary vasculature perfused with $10 \mathrm{ml}$ of heparinized PBS $(40 \mathrm{U} / \mathrm{ml})$ via the pulmonary artery. Lungs were inflated with formalin under set constant gravitational pressure $\left(20 \mathrm{~cm} \mathrm{H}_{2} \mathrm{O}\right)$, and the lungs removed en bloc and fixed in $10 \mathrm{ml}$ of formalin. Fixed tissues were embedded in paraffin wax prior to sections $5 \mu \mathrm{m}$ thick being cut, rehydrated in graded alcohol and stained by Masson's trichrome. Severity of lung fibrosis was quantified through Ashcroft scoring ${ }^{21}$ of Masson's trichrome stained tissue sections.

\section{Immunohistochemistry}

Five-micrometer-thick lung tissue sections were deparaffinized in xylene and rehydrated in graded ethanol. Antigen retrieval was performed via heating sections in $10 \mathrm{~mm}$ sodium citrate buffer (pH6.0) in a microwave for $10 \mathrm{~min}$. Subsequently, endogenous peroxidase activity was blocked by incubating sections in $3 \% \mathrm{H}_{2} \mathrm{O}_{2}$ in methanol for $30 \mathrm{~min}$. Nonspecific antibody binding was blocked using goat serum (Sigma Aldrich) for $30 \mathrm{~min}$, prior to incubation with human anti-mouse collagen type I (ab292) $(2 \mu \mathrm{g} / \mathrm{ml})$, III (ab7778) $(1 \mu \mathrm{g} / \mathrm{ml})$, IV (ab6586) $(0.5 \mu \mathrm{g} / \mathrm{ml})$, VI (6588) $(0.5 \mu \mathrm{g} / \mathrm{ml})$ (Abcam, Cambridge, UK), or rabbit anti-mouse PhosphoSmad2 (\#3101) (1:2000 dilution) primary antibody (Cell Signaling Technology, Boston, MA, USA) overnight at $4{ }^{\circ} \mathrm{C}$. Sections were then incubated with a biotin-conjugated secondary antibody. Biotin-conjugated secondary antibody was detected through incubation with an avidin/biotinylated enzyme complex solution (Vector Laboratories) for $30 \mathrm{~min}$; color development was performed using 3,3'-diaminobenzidine tetrahydrochloride (Sigma Aldrich). Sections were counterstained using Mayer's hematoxylin and visualized under a Nikon 90i light microscope. The total area of collagen I, III, IV, and VI, positively stained tissue, in fibrotic lesions $\geqslant 7500 \mu \mathrm{m}^{2}$, was measured using NIS-Elements (Nikon instruments). A single cross section from all five lung lobes, per mouse, were analyzed and total area of positively stained collagen was calculated as a percentage of total lung tissue area.

\section{Analysis of Lung Collagen Content}

Lungs were removed and immersed immediately in liquid nitrogen prior to storage at $-80^{\circ} \mathrm{C}$. Lung tissue was ground to a fine powder under liquid nitrogen. Ground lung tissue was mixed in $1 \mathrm{ml}$ distilled water on ice in Pyrex tubes and incubated in $125 \mu \mathrm{l} 50 \%$ TCA (Sigma Aldrich) at $4{ }^{\circ} \mathrm{C}$ for $20 \mathrm{~min}$. Samples were centrifuged at $528 \mathrm{~g}$ for $10 \mathrm{~min}$ at $4{ }^{\circ} \mathrm{C}$. 
The lungs were then hydrolyzed in $1 \mathrm{ml}$ of $12 \mathrm{~N}$ HCL overnight at $110^{\circ} \mathrm{C}$ prior to reconstitution in $2 \mathrm{ml}$ of distilled water. In total, $200 \mu \mathrm{l}$ of hydrozylate, or hydroxyproline standard (Sigma Aldrich), were oxidized in $500 \mu \mathrm{l}$ chloramine $\mathrm{T}$ (Sigma Aldrich) for $20 \mathrm{~min}$ at room temperature (RT) prior to the addition of $500 \mu \mathrm{l}$ of Ehrlich's solution (Sigma Aldrich) and incubation at $65^{\circ} \mathrm{C}$ for $15 \mathrm{~min}$. Samples were then incubated at RT for $2 \mathrm{~h}$ prior to colorimetric analysis at an absorbance of $550 \mathrm{~nm}$. Hydroxyproline per $\mathrm{mg}$ of lung tissue was calculated against a standard curve.

\section{RT-PCR}

RNA was isolated from frozen lung tissue using a TRIzol-based extraction protocol. RNA $(2 \mu \mathrm{g})$ was reverse transcribed using moloney murine leukemia virus reverse transcriptase (Promega) to produce cDNA for real time PCR (RT-PCR) analysis. Primers used: Slpi forward-5'-GCTGTGAGGGTATATGTGGGAAA-3' and reverse- $5^{\prime}$-CGCCAATGTCAGGGATCAG-3', Mmp-9 forward-5'-TGAATCAGCTGGCTTTTGTG-3' and reverse- $5^{\prime}$ GTGGATAGCTCGGTGGTGTT-3', Coll $\alpha 1$ forward-5'-GGA GGGCGAGTGCTGTGCTTT- $3^{\prime}$ and reverse- $5^{\prime}$-GGGACCAG GAGGACCAGGAAGT-3', Col3 $\alpha 1$ forward-5'-AGGTACCGA TTTGAACAGGCT- $3^{\prime}$ and reverse- $5^{\prime}$-TTTGCAGCCTGGGCT CATTT-3', Col4 $\alpha 1$ forward-5'-AAGCCCATTCCTCCAACTG

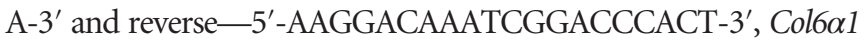
forward-5'-GATGAGGGTGAAGTGGGAGA-3' and reverse5'-CAGCACGAAGAGGATGTCAA-3'. Amplification was performed using an MXPro3000 QPCR system (Stratagene). Kappa Taq polymerase (Kapa Biosystems) was activated at $95^{\circ} \mathrm{C}$ for 3 min followed by 40 cycles of denaturing, annealing and extension $\left(95^{\circ} \mathrm{C} 30 \mathrm{~s}, 60^{\circ} \mathrm{C} 30 \mathrm{~s}\right.$, and $72^{\circ} \mathrm{C} 20 \mathrm{~s}$, respectively). Annealing was conducted at $59^{\circ} \mathrm{C}$ for Col6al. Ct values were standardized to a housekeeper gene, hypoxanthine-guanine phosphoribosyltransferase: forward-5'-TGAAAGACTTGCTCGAGA TGTCA- $3^{\prime}$ and reverse- $5^{\prime}$-CCAGCAGGTCAGCAAAGAAC T-3'. Amplification of a single DNA product was confirmed by melting curve analysis. Data expressed as mean relative expression using the $\Delta \Delta C_{\mathrm{t}}$ equation as described previously. ${ }^{22}$

\section{Zymography}

Matrix metalloproteinase (MMP)-2 and MMP-9 activity was assessed via gelatin zymography. Ten microliter of bronchoalveolar lavage supernatant was diluted 1:1 in Tris-Gly SDS zymogram samples buffer (Invitrogen), and incubated at $21^{\circ} \mathrm{C}$ for $10 \mathrm{~min}$. Fifteen microliter of sample (or standard) was loaded onto zymogram precast gelatin gels, $10 \%$ Tris-Glycine gel with $0.1 \%$ gelatin substrate (Invitrogen) and subjected to electrophoresis at constant voltage of $125 \mathrm{~V}$ for $100 \mathrm{~min}$. Gels were incubated at RT for $60 \mathrm{~min}$ in $100 \mathrm{ml}$ of zymogram renaturing buffer, 2.5\% Triton X-100 (Invitrogen), to recover enzymatic activity. Gels were then incubated in zymogram-developing buffer, $50 \mathrm{mM}$ Tris- $\mathrm{HCl}, \mathrm{pH} 7.5$, $200 \mathrm{mM} \mathrm{NaCl}, 5 \mathrm{mM} \mathrm{CaCl} 2$ (Invitrogen) for $1 \mathrm{~h}$ prior to overnight incubation at $37^{\circ} \mathrm{C}$. Gels were then stained with coomassie blue-stain (Coomassie blue $0.1 \%(\mathrm{w} / \mathrm{v})$ in $\mathrm{H}_{2} \mathrm{O}$
$50 \%(\mathrm{v} / \mathrm{v})$, methanol $40 \%(\mathrm{v} / \mathrm{v})$, and acetic acid 10\% (v/v)) for $60 \mathrm{~min}$ before destaining in the same solution without the dye for $30 \mathrm{~min}$. Bands were then visualized by image analysis (Gel Doc, Syngene, Cambridge, UK) and densitometry determined using Image $\mathrm{J}$ software. Band densities were standardized to the mean density of two control samples, per gel, to allow comparisons between multiple gels. All gels were run in parallel.

\section{Macrophage TGF- $\beta$ Activity}

Nuclear fraction of bronchoalveolar lavage cells was extracted, 28 days post bleomycin treatment, using a nuclear extraction kit (Active-motif). In brief, lungs were lavaged with a total of $4.5 \mathrm{ml}$ of PBS-containing phosphatase inhibitor (Activemotif). Cells were pelleted via centrifugation at $528 \mathrm{~g}$ for $10 \min \left(4^{\circ} \mathrm{C}\right)$ and the supernatant was removed and stored at $-80^{\circ} \mathrm{C}$. Total cell pellets were resuspended in $250 \mu \mathrm{l}$ of hypotonic lysis buffer and incubated on ice for $15 \mathrm{~min}$. In total, $12.5 \mu \mathrm{l}$ of detergent (Active-motif) was added and the samples were vortexed for $10 \mathrm{~s}$ prior to centrifugation for $1 \mathrm{~min}$ at $14000 \mathrm{~g}\left(4^{\circ} \mathrm{C}\right)$ and resuspended in $25 \mu \mathrm{l}$ of lysis buffer (Active-motif). Samples were vortexed and centrifuged for $1 \mathrm{~min}$ at $14000 \mathrm{~g}\left(4^{\circ} \mathrm{C}\right)$ and the nuclear fraction removed for pSmad2 assessment using the PathScan Total Smad2 Sandwich ELISA Kit (Cell Signaling Technology). Ten micogram of total cell nuclear protein was assayed from each sample.

\section{Neutrophil Elastase Activity}

Twenty-eight days post bleomycin treatment, lungs were excised and snap-frozen in liquid nitrogen prior to storage at $-80^{\circ} \mathrm{C}$. Lung tissue was ground to a fine powder under liquid nitrogen and resuspended in $1 \mathrm{ml} 0.1 \mathrm{M}$ Tris $\mathrm{pH}$ 7.4. After three cycles of freezing and thawing, the lung lysates were centrifuged at $20800 \mathrm{x}$ g for $30 \mathrm{~min}$ at $4{ }^{\circ} \mathrm{C}$. NE activity was measured in lung homogenate using the synthetic fluorescent, MeOSu-AAPV-AMC substrate (Santa-Cruz, Dallas, TX, USA). Lung homogenate $(1.5 \mathrm{mg} / \mathrm{ml}$ protein concentration) was incubated with MeOSu-AAPV-AMC $(0.1 \mathrm{nM}$ final concentration) in $0.1 \mathrm{M}$ Tris pH 7 (total volume $250 \mu \mathrm{l}$ ) in a black bottom/wall 96-well plate and the amount of NE released fluorescent product was determined at $2 \mathrm{~h}$. Plates were read using excitation $355 \mathrm{~nm}$ and $460 \mathrm{~nm}$ emission wavelengths; background fluorescence of the substrate only was subtracted from each sample.

\section{Statistical Analysis}

All data are reported as mean \pm s.e.m. of $n$ observations. Student's $t$-test was used for comparison between two groups. Two-way ANOVA was used for comparison of multiple data sets. $P$-values $<0.05$ were considered significant. All statistical analysis was performed using GraphPad Prism (v6.01, La Jolla, CA, USA). 
a
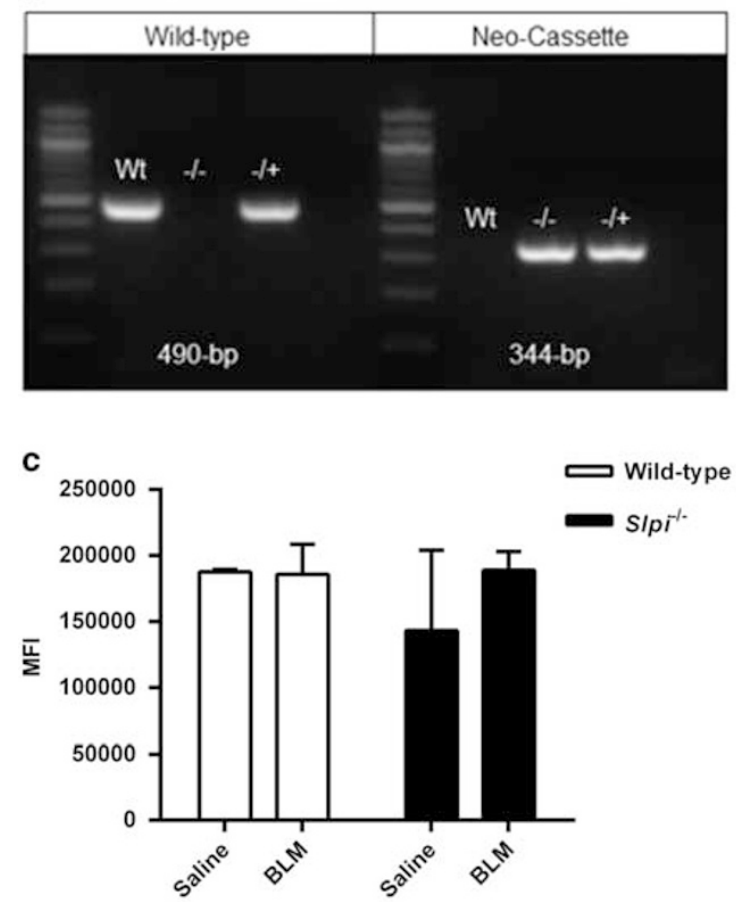

e

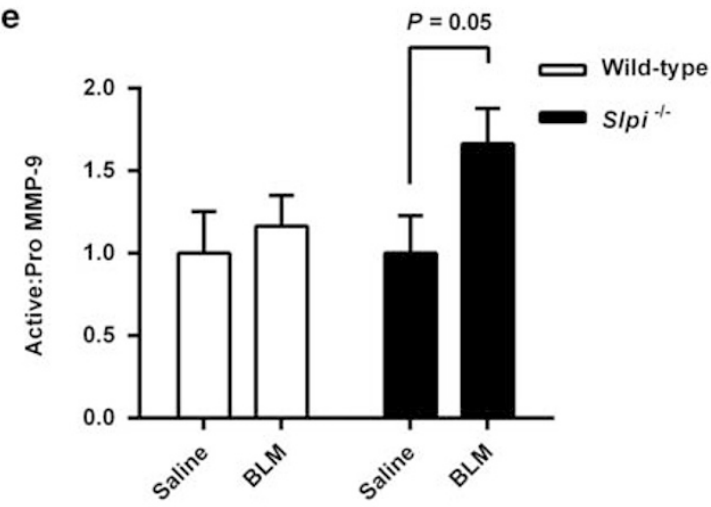

b

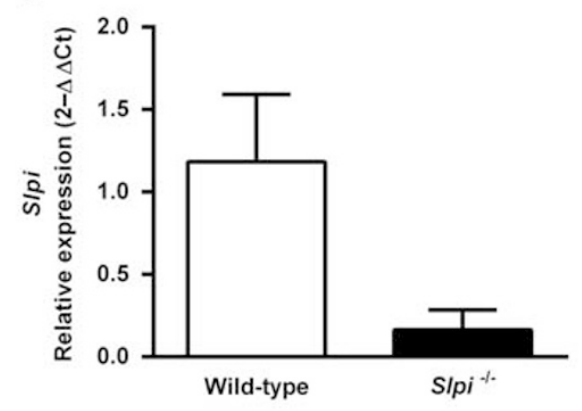

d

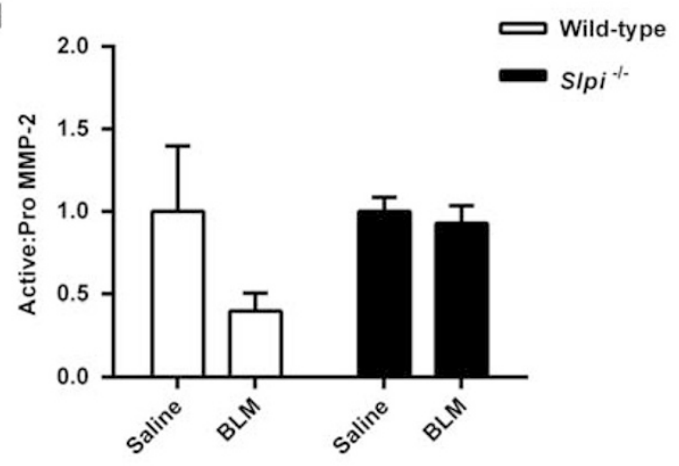

f

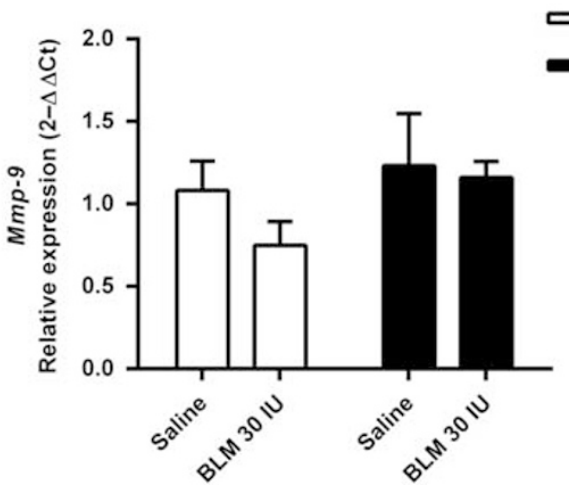

Figure $1 \mathrm{Slpi}^{-1-}$ mice do not express Slpi mRNA and have enhanced MMP-9 activity in the lung. (a) PCR confirmation of presence of wild-type 490-bp fragment and absence of Neo-cassette fragment 344-bp in wild-type animals $(\mathrm{Wt})$ and absence of wild-type fragment and presence of Neo-cassette in Slpi $i^{-1}$ animals $(-/-)$. Both wild-type and Neo-cassette fragments were present in heterozygous animal (-/+). (b) Slpi gene expression in lung homogenates from wild-type and Slpi ${ }^{-1-}$ animals. Data expressed as mean relative expression $\left(\Delta \Delta C_{t}\right) \pm$ s.e.m.; $n=3$. (c) Neutrophil elastase activity was assessed in lung homogenate 28 days post bleomycin treatment. Data expressed as mean fluorescence intensity (MFI) \pm s.e.m.; $n \geqslant 2$. (d) Ratio of pro and active forms of MMP-2 in BAL supernatant from wild-type and Slpi ${ }^{-1-}$ mice and (e) ratio of pro and active forms of MMP-9 in BAL supernatant from wild-type and Slpi ${ }^{-1-}$ mice. Mean \pm s.e.m.; $n=8$. (f) $M m p-9$ mRNA levels in lung homogenates 28 days post bleomycin (BLM). Data expressed as mean relative expression $\left(\Delta \Delta C_{t}\right) \pm$ s.e.m.; $n=8$.

\section{Results}

\section{SIpi Gene Deletion Promotes a Proteolytic Environment in the Lung}

The genotype of $S l p i^{-1-}$ mice was confirmed by PCR (Figure 1a). Slpi mRNA was analyzed in lung tissue by quantitative RT-PCR to confirm that $S l p i^{-1-}$ mice had Slpi deficiency in the lung (Figure 1b). To determine whether Slpi deficiency altered serine proteinase activity NE activity was measured 28 days post bleomycin, but no differences were detected regardless of genotype or injury (Figure 1c). Serine proteases can activate both MMP-2 and MMP-9, ${ }^{23,24}$ therefore the ratio of pro-MMP-2 and MMP-9 and active MMP-2 and MMP-9 was determined 28 days post bleomycin (Figures 1d and e, respectively; gelatin zymography gel images available in Supplementary Figures S1a, b and c). There was no difference between levels of active MMP-2 and MMP-9 in saline exposed mice of either genotype. However, following bleomycin exposure, there was no increase in active MMP-2 


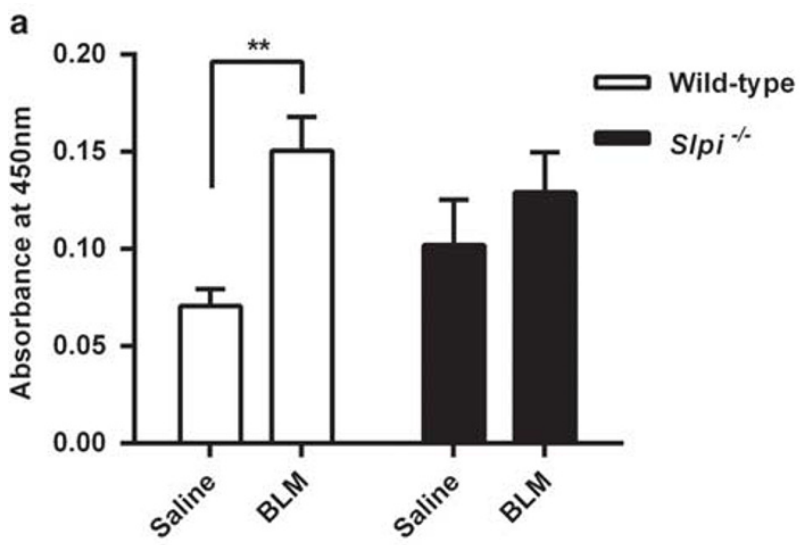

b

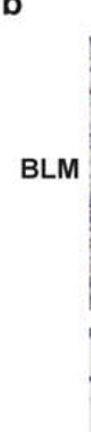

Saline
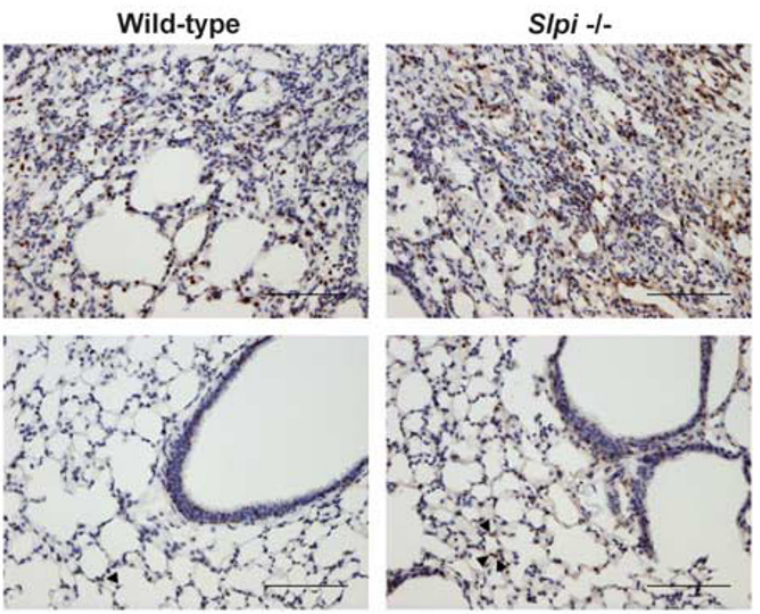

d

C

Wild-type
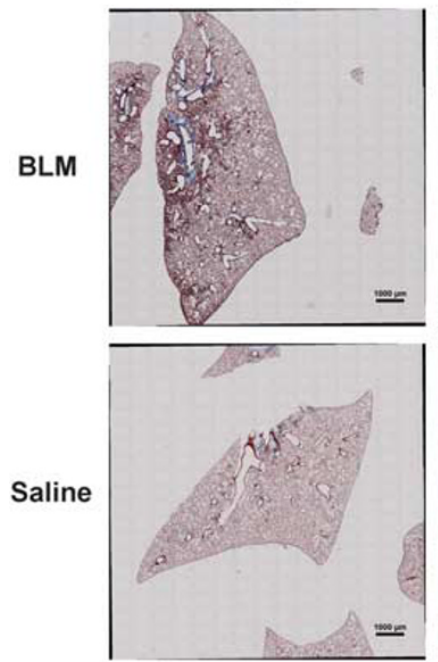

SIpi -l-
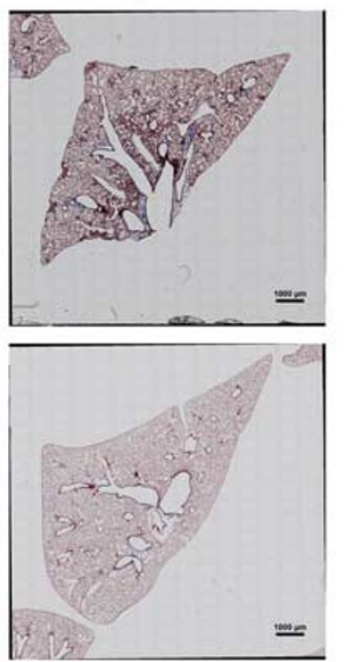

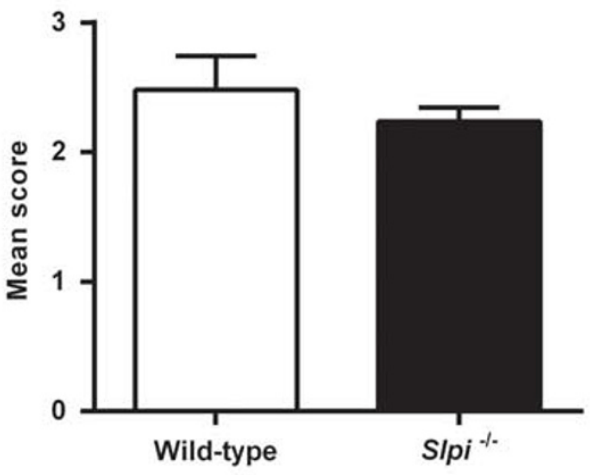

Figure 2 The effect of Slpi deletion on alveolar macrophage TGF- $\beta$ activation and pulmonary fibrosis. (a) pSmad2 levels in nuclear extracts from BAL cells from Slpi $i^{-1-}$ and wild-type mice 28 days post bleomycin (BLM). Data expressed as mean absorbance at $450 \mathrm{~nm} \pm$ s.e.m.; $n \geqslant 5 ;{ }^{* *} P<0.005$ BLM vs saline. (b) pSmad2 immunohistochemistry. Representative images captured using original magnification $\times 20$, scale bars $100 \mu \mathrm{m}$. Arrowheads identify positively stained cells within a single, representative, alveolus in saline-treated mice only; there is just one arrowhead in the wild-type mouse and three in the Slpi ${ }^{-1-}$ mouse. (c) Masson's trichrome staining 28 days post BLM treatment. Representative, whole lung lobe, cross sections. (d) Quantitative assessment of trichrome staining. Data expressed as mean Ashcroft Score \pm s.e.m.; $n \geqslant 3$.

(Figure 1d) but an increase in active MMP-9 in bleomycin exposed $S l p i^{-1-}$ mice, compared with either saline or wildtype controls $(P=0.05)$, was detected (Figure 1e). The effects of SLPI on active MMP-9 levels were not due to enhanced MMP-9 expression because no differences in Mmp-9 mRNA levels were found between wild-type and $\mathrm{Slpi}^{-1-}$ mice exposed to saline or bleomycin 28 days post bleomycin (Figure 1f).

\section{Bleomycin-Induced Lung Fibrosis Does Not Enhance Alveolar TGF- $\beta$ Activation In SIpi ${ }^{-1-}$ Mice, Although They Still Develop Pulmonary Fibrosis}

To determine the effect of Slpi gene deletion on the development of pulmonary fibrosis, macrophage TGF- $\beta$ activation and lung histology in Slpi $i^{-1-}$ mice were evaluated. Levels of nuclear pSmad2 from alveolar macrophages were significantly increased in wild-type mice 28 days post bleomycin exposure compared with saline controls $(P=0.003$; Figure $2 \mathrm{a})$. In contrast, there was a trend toward increased basal pSmad2 in Slpi ${ }^{-1-}$ mice but this was not significantly increased following bleomycin exposure (Figure 2a). Global tissue TGF- $\beta$ activation was measured by $\mathrm{pSmad} 2$ immunostaining. In saline instilled mice there was evidence of increased pSmad2 staining in $S l p i^{-1-}$ mice around both the alveoli and larger airways (Figure 2b). Bleomycin treatment increased pSmad2 staining in both wild-type and Slpi ${ }^{-1-}$ mice, primarily at areas of injury and fibroproliferation. Examination of lung sections from both wild-type, and 
Slpi $i^{-1-}$, mice demonstrated areas of severe fibrosis following bleomycin exposure compared with saline controls (Figure 2c). The extent of lung fibrosis was quantified using a modified Ashcroft score and revealed there was no difference in the severity of fibrosis in wild-type mice compared with $S l p i^{-1-}$ animals (Figure 2d).

\section{SIpi Gene Deletion Exacerbates Bleomycin-Induced Weight Loss but Does Not Alter Hydroxyproline Deposition}

It was noted that $S l p i^{-1-}$ mice lost more weight than wildtype mice during the acute lung injury phase of bleomycininduced lung injury (Figure 3a). Weight loss was significantly greater in $\mathrm{Slpi}^{-1-}$ mice 4 days post bleomycin treatment $(P=0.02)$ and peaked 5 days post bleomycin (Figure 3a). Therefore, the effect of Slpi gene deletion on hydroxyproline levels during the inflammatory, early and late fibrotic phases of fibrogenesis following bleomycin exposure was assessed (7, 14, and 28 days post bleomycin, respectively). There was a progressive increase in hydroxyproline deposition during all phases of fibrogenesis in wild-type mice that reached statistical significance 28 days following bleomycin exposure $(P=<0.0001$ Figure 3b). There was also an increase in hydroxyproline in $S l p i^{-1-}$ mice 28 days following bleomycin although the levels appeared marginally, but non-significantly, lower at each time point than in wild-type mice (Figure 3b).

\section{SIpi Gene Deletion Impairs Col1a1, Col3a1, And Col4a1 Gene Expression During Fibrogenesis but Promotes An Early Transient Increase in Col6a1 Gene Expression}

Sustained expression of Col1 $\alpha 1$ and Col4 $\alpha 1$ was only observed in wild-type mice, whereas in Slpi $i^{-1-}$ mice there was a small initial rise in Coll $\alpha 1$ and Col4 $\alpha 1$ gene expression but this did not increase during the fibrotic phase of the model and 28 days post bleomycin levels of Coll $\alpha 1$ gene expression were significantly greater in wild-type mice compared with $S l p i^{-1-}$ animals $(P=0.0124)$ (Figure 4a). In wild-type animals, the expression of Col3 $\alpha 1$ mRNA was significantly increased 28 days post bleomycin and, again, there was no increase in $\mathrm{Slpi}^{-1-}$ animals. However, an early and transient increase in Col6 1 mRNA 7 days following injury was observed in Slpi ${ }^{-1-}$ animals $(P=0.011)$ (Figure $\left.4 \mathrm{a}\right)$. Lung tissue sections, taken 28 days post bleomycin instillation, were stained for collagen type I, III, IV, and VI (Figure 4b). Collagen type I and VI positively stained tissue, in fibrotic lesions $\geq 7500 \mu \mathrm{m}^{2}$, was evaluated. Despite differences in gene expression between wild-type and $\mathrm{Slpi}^{-1-}$ mice there are no differences in collagen type I and VI composition of fibrotic lesions (Figure 4c).

\section{Discussion}

SLPI is an inhibitor of protease activity and deletion of Slpi in mice has been associated with increased elastase activity, enhanced TGF- $\beta$ activity, prolonged wound healing and
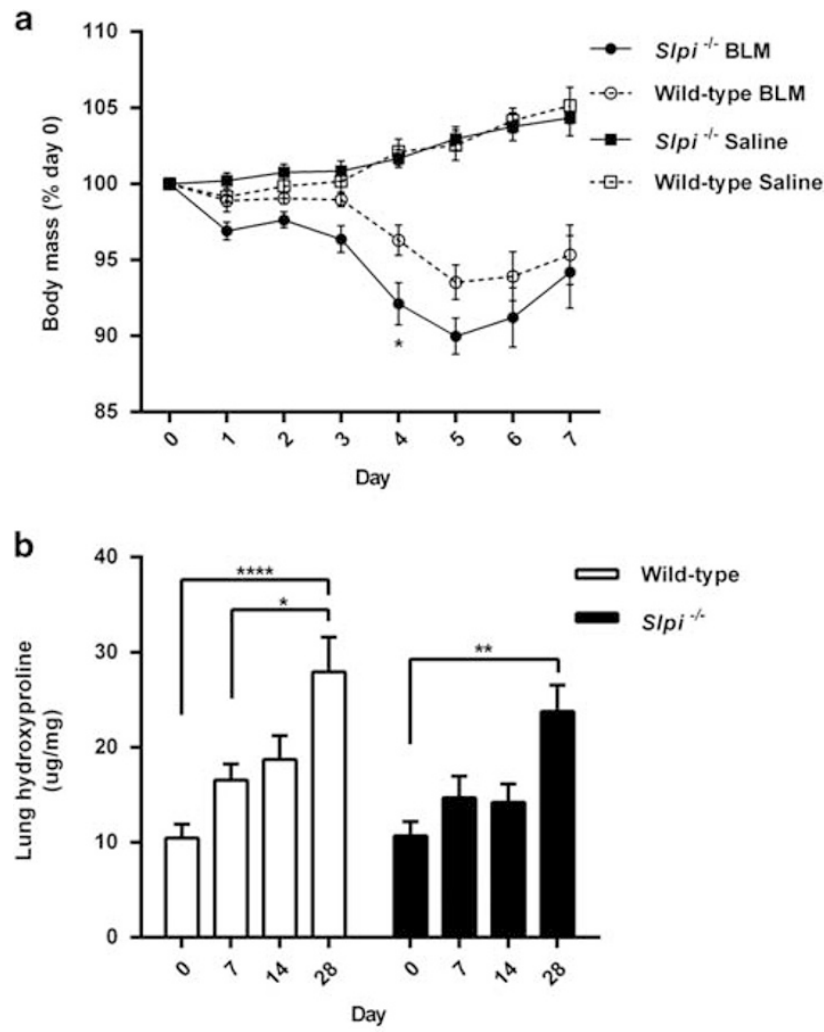

Figure 3 The effect of Slpi deletion on weight loss and lung hydroxyproline levels. (a) Body weight during the acute lung injury phase of the model in $\mathrm{Slpi}^{-1-}$ compared with wild-type mice. Data expressed as mean percentage of initial (day 0 ) body mass (\%) \pm s.e.m.; $n \geqslant 9$; Significant difference determined at day $4 * P<0.05 \mathrm{Slpi}^{-1-}$ vs wild-type post bleomycin (BLM). (b) Lung hydroxyproline levels were assessed at 0 , 7,14 , and 28 days following BLM in Slpi ${ }^{-1-}$ vs wild-type mice. Data expressed as the amount of hydroxyproline per $\mathrm{mg}$ of lung tissue $(\mu \mathrm{g} / \mathrm{mg}) \pm$ s.e.m.; $n \geq 8$. ${ }^{*} P<0.05,{ }^{* *} P<0.01$, and ${ }^{* * *} P<0.0001$.

increased inflammatory cell influx into wounds. ${ }^{19,20} \mathrm{We}$ therefore hypothesized Slpi ${ }^{-1-}$ mice would have enhanced lung injury and fibrosis compared with wild-type controls. Surprisingly, despite evidence of enhanced metalloproteinase activity, more severe injury and increased Col6 $\alpha 1$ gene expression at early time points, we did not observe worsening fibrosis; indeed a trend toward reduced fibrosis was observed. Furthermore, we did not detect enhanced alveolar TGF- $\beta$ activity following injury.

Assessment of alveolar TGF- $\beta$ activation can be achieved by measuring nuclear Smad2 phosphorylation, a process specific to TGF- $\beta$ signaling, in alveolar macrophages, whereas global TGF- $\beta$ activity can be assessed by immunohistochemistry. Alveolar activation of TGF- $\beta$ in normal lungs is primarily mediated via the $\alpha \mathrm{v} \beta 6$ integrin..$^{25,26}$ Wild-type mice had a significant increase in phosphorylation of macrophage Smad2 following bleomycin supporting the important role of $\alpha \mathrm{v} \beta 6$ integrin-mediated TGF- $\beta$ activation in this model. ${ }^{26}$ Although there was a trend toward increased basal alveolar TGF- $\beta$ activity in $S l p i^{-1-}$ mice, there was no significant increase 
a

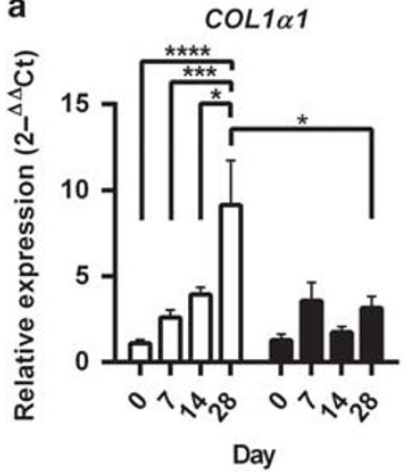

b

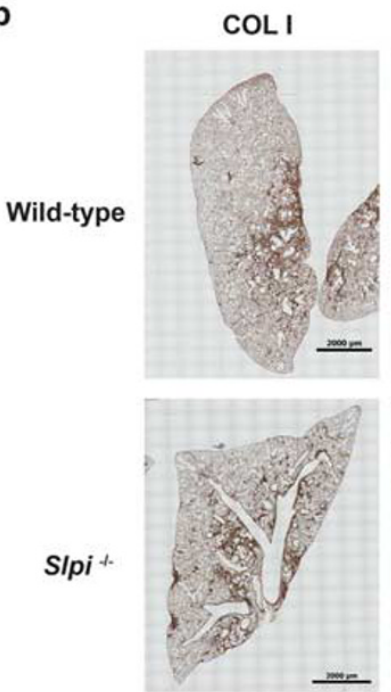

$\operatorname{COL} 3 \alpha 1$

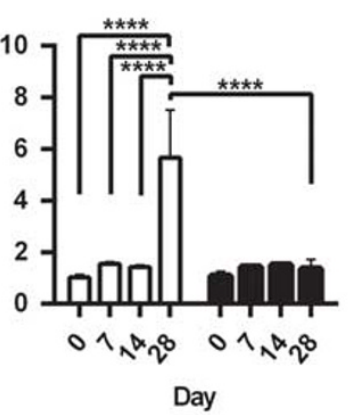

Col $4 \alpha 1$
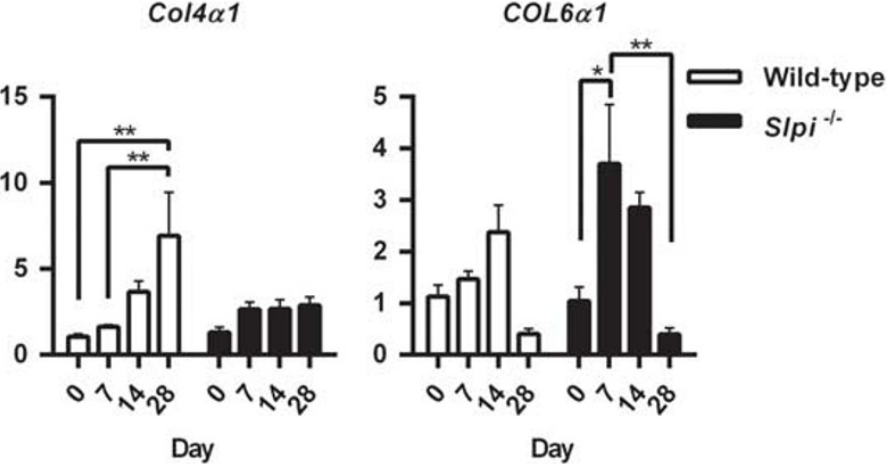

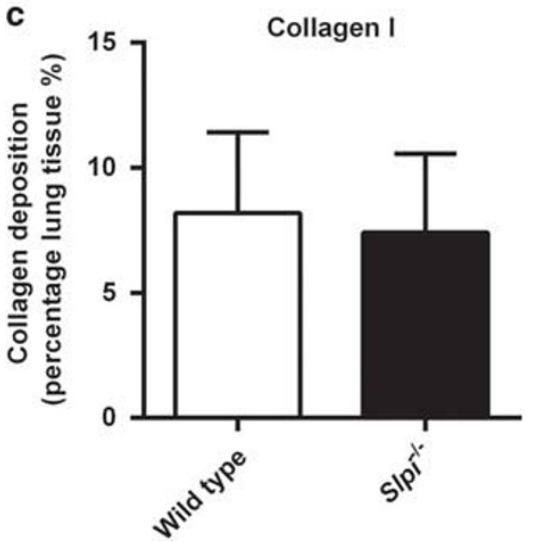

COL III
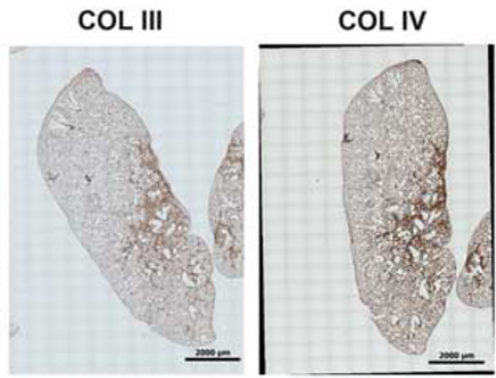

COL VI
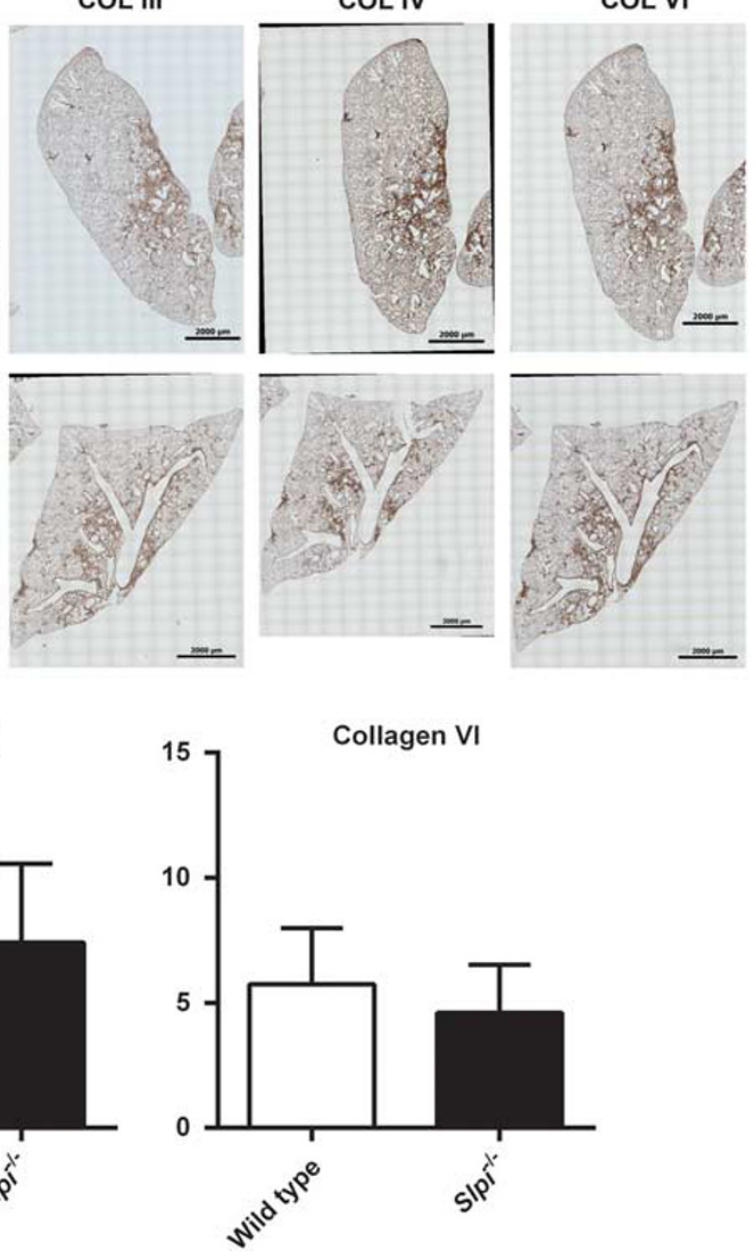

Figure 4 The effect of Slpi deletion on subtype-specific collagen synthesis and deposition. (a) Col1a1, Col3a1, Col4a1, and Col6a1 gene expression 0, 7, 14 , and 28 days post bleomycin (BLM). Data expressed as mean relative expression $\left(\Delta \Delta C_{t}\right) \pm$ s.e.m.; $n \geqslant 8,{ }^{*} P<0.05$, ${ }^{* *} P<0.01,{ }^{* * *} P<0.001$, and ${ }^{* * * *}$ $P<0.0001$. (b) $5 \mu \mathrm{m}$ thick tissue sections from BLM treated wild-type and SIpi $i^{-1}$ mice were stained with anti-collagen I, anti-collagen III, anti-collagen IV, and anti-collagen VI. Low-magnification $(\times 10)$ images were stitched together to visualize whole lung lobes. Images are representative of $n \geqslant 3$ animals. (c) Collagen I and VI deposition was measured in lung tissue sections. Data expressed as mean percentage of total lung tissue comprised of fibrotic lesions $\geqslant 7500 \mu \mathrm{m}^{2}$ containing collagen I and collagen VI (\%) \pm s.e.m.; $n \geqslant 3$.

above these higher basal levels following bleomycin-induced lung injury. It is known that tethering of the latent TGF- $\beta$ complex to a stiff extracellular matrix is crucial for the force generation required for $\alpha v \beta 6$ integrin-mediated TGF- $\beta$ activation. ${ }^{27,28}$ It is therefore possible that increased matrix degradation associated with reduced SLPI prevents sufficient force generation required for this mechanism of TGF- $\beta$ activation. Increased pSmad 2 was similarly observed in the 
alveoli of Slpi $i^{-1-}$ mice at baseline consistent with the data from alveolar macrophages and data from previously published studies. ${ }^{19}$ However, even in Slpi ${ }^{-1-}$ mice there was an increase in $\mathrm{pSmad} 2$ in the fibroproliferative lesions of mice following bleomycin. Moreover, pSmad2 levels in bleomycin treated wild-type, and Slpi ${ }^{-1-}$ mice, were not different using either assessment of TGF- $\beta$ activity suggesting that the effect of bleomycin on TGF- $\beta$ activation, especially in mesenchymal cells, is independent of SLPI, which may explain the development of lung fibrosis in animals of either genotype.

No difference in NE activity in lung homogenates from mice of either genotype or level of injury was detected. Although this may reflect the timing of the assay after the resolution of inflammation, at the peak of the fibroproliferative phase of the bleomycin model, it may also reflect the importance of other proteinases, such as the lysosomal cysteine proteinase cathepsin K. Cathepsin $\mathrm{K}$ has potent collagenolytic activity and transgenic mice over-expressing it have reduced lung fibrosis. ${ }^{29}$ Similarly, $\mathrm{Ctsk}^{-1-}$ mice have increased bleomycin-induced pulmonary fibrosis, ${ }^{30}$ and furthermore cathepsin $\mathrm{K}$ inhibits TGF- $\beta$ generation. ${ }^{31}$ In clinical studies of osteoporosis a small number of patients treated with the cathepsin $\mathrm{K}$ inhibitor balicatib developed skin fibrosis $^{32}$ consistent with the pro-fibrotic effect of cysteine proteinases.

Previous studies have suggested that SLPI can inhibit MMP-9 expression, however our studies did not demonstrate any difference in total $\mathrm{Mmp}-9$ levels at the transcriptional level between wild-type and Slpi ${ }^{-1-}$ mice suggesting that SLPI is required predominately to regulate post-translational modification of MMP-9 in vivo, consistent with previous studies assessing oral wounds ${ }^{19,20}$ and the observation that NE can activate MMP-9 in vivo. ${ }^{24}$ Increased MMP-9 has been associated with both $\mathrm{IPF}^{33}$ and experimental models of lung fibrosis. ${ }^{34-36}$ Increased MMP-9 activity promotes fibrosis through proteolysis of the alveolar epithelial basement membrane, particularly collagen IV, resulting in impaired re-epithelialization and fibroblast migration into the alveolar space, ${ }^{34-36}$ and this could in part explain the development of pulmonary fibrosis in $\mathrm{Slpi}^{-1-}$ mice.

Collagen deposition during wound healing is tightly regulated and dysregulation results in a fibroproliferative state. ${ }^{37}$ Our data show that there are sustained increases in Coll $\alpha 1$ and Col4 $\alpha 1$, in wild-type animals in the first 4 weeks following bleomycin-induced fibrosis. However, no sustained

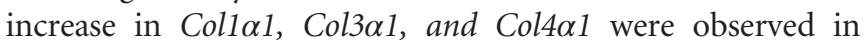
Slpi $i^{-1-}$ mice, suggesting that SLPI is somehow associated with their expression in the lungs. Despite the lack of increased gene expression, there were similar levels of collagen I, III, IV, and VI deposition in the lung, assessed by immunohistochemistry. These data suggest that in $S l p i^{-/-}$mice, in contrast with wild-type mice, collagen deposition may be less dependent on collagen gene expression but could occur as a result of increased post-translational modification in a protease rich environment. Collagen VI is deposited during wound healing and is transient and precedes colocalized, collagen I deposition ${ }^{38,39}$ and this is consistent with our data. Interestingly, in contrast with expression of Col1 $\alpha 1$, Col3 $\alpha 1$, and Col4 $\alpha 1$, our data suggest that Col6 $\alpha 1$ gene expression is independently regulated.

In summary, these data suggest that $\mathrm{Slpi}^{-1-}$ mice have enhanced metalloprotease activity and lung injury following bleomycin but they lack an exaggerated pulmonary fibrotic response in the first month following injury. Furthermore, SLPI deficiency impairs Coll $\alpha 1$, Col3 $\alpha 1$, and Col4 $\alpha 1$ gene expression following injury despite collagen deposition. We hypothesize that a metalloprotease-rich environment promotes post-translational processing and collagen deposition resulting in lung fibrosis.

Supplementary Information accompanies the paper on the Laboratory Investigation website (http://www.laboratoryinvestigation.org)

\section{ACKNOWLEDGMENTS}

This work was funded by a Wellcome Trust Research Grant Number WT085350MA. Slpi ${ }^{-1}$ mice on a B6D2F1 background were kindly donated by Sharon Wahl. This research was funded by the Wellcome Trust (project grant number 085350), and in part supported by the Intramural Research Program of the NIH, National Institute of Dental and Craniofacial Research.

\section{DISCLOSURE/CONFLICT OF INTEREST}

Dr Gisli Jenkins has Sponsored Research Grants from GlaxoSmithKline, Novartis and Biogen Idec. He has undertaken consultancy work for GlaxoSmithKline, Boehringer Ingelheim, PharmAkea Therapeutics, Roche, Biogen Idec and Intermune and has received lecture fees from Medlmmune.

1. Maher TM, Wells AU, Laurent GJ. Idiopathic pulmonary fibrosis: multiple causes and multiple mechanisms? Eur Respir J 2007;30: 835-839.

2. Gauldie J, Jordana M, Cox G. Cytokines and pulmonary fibrosis. Thorax 1993;48:931-935.

3. Selman M, King TE, Pardo A. Idiopathic pulmonary fibrosis: prevailing and evolving hypotheses about its pathogenesis and implications for therapy. Ann Intern Med 2001;134:136-151.

4. Kinder BW, Brown KK, Schwarz Ml et al. Baseline BAL neutrophilia predicts early mortality in idiopathic pulmonary fibrosis. Chest 2008;133:226-232.

5. Pham CT. Neutrophil serine proteases: specific regulators of inflammation. Nat Rev Immunol 2006;6:541-550.

6. Chua F, Laurent GJ. Neutrophil elastase: mediator of extracellular matrix destruction and accumulation. Proc Am Thorac Soc 2006;3: 424-427.

7. Chua F, Dunsmore SE, Clingen PH et al. Mice lacking neutrophil elastase are resistant to bleomycin-induced pulmonary fibrosis. Am J Pathol 2007;170:65-74.

8. Sakashita A, Nishimura Y, Nishiuma T et al. Neutrophil elastase inhibitor (sivelestat) attenuates subsequent ventilator-induced lung injury in mice. Eur J Pharmacol 2007;571:62-71.

9. Taooka $Y$, Maeda A, Hiyama $\mathrm{K}$ et al. Effects of neutrophil elastase inhibitor on bleomycin-induced pulmonary fibrosis in mice. Am J Respir Crit Care Med 1997;156:260-265.

10. Obayashi Y, Yamadori I, Fujita J et al. The role of neutrophils in the pathogenesis of idiopathic pulmonary fibrosis. Chest 1997;112: 1338-1343.

11. Hojo S, Fujita J, Yoshinouchi T et al. Hepatocyte growth factor and neutrophil elastase in idiopathic pulmonary fibrosis. Respir Med 1997;91:511-516.

12. Wang $Y$, Shiota $N$, Leskinen MJ et al. Mast cell chymase inhibits smooth muscle cell growth and collagen expression in vitro: transforming 
growth factor-beta1-dependent and -independent effects. Arterioscler Thromb Vasc Biol 2001;21:1928-1933.

13. Taipale J, Koli K, Keski-Oja J. Release of transforming growth factor-beta 1 from the pericellular matrix of cultured fibroblasts and fibrosarcoma cells by plasmin and thrombin. J Biol Chem 1992;267:25378-25384.

14. Lyons RM, Gentry LE, Purchio AF et al. Mechanism of activation of latent recombinant transforming growth factor beta 1 by plasmin. J Cell Biol 1990;110:1361-1367.

15. Jenkins RG, Su X, Su G et al. Ligation of protease-activated receptor 1 enhances alpha(v)beta6 integrin-dependent TGF-beta activation and promotes acute lung injury. J Clin Invest 2006;116:1606-1614.

16. Scotton CJ, Krupiczojc MA, Konigshoff $M$ et al. Increased local expression of coagulation factor $\mathrm{X}$ contributes to the fibrotic response in human and murine lung injury. J Clin Invest 2009;119:2550-2563.

17. Eisenberg SP, Hale KK, Heimdal $P$ et al. Location of the proteaseinhibitory region of secretory leukocyte protease inhibitor. J Biol Chem 1990;265:7976-7981.

18. Abe $\mathrm{T}$, Kobayashi $\mathrm{N}$, Yoshimura $\mathrm{K}$ et al. Expression of the secretory leukoprotease inhibitor gene in epithelial cells. J Clin Invest 1991;87: 2207-2215.

19. Ashcroft GS, Lei K, Jin W et al. Secretory leukocyte protease inhibitor mediates non-redundant functions necessary for normal wound healing. Nat Med 2000;6:1147-1153.

20. Angelov N, Moutsopoulos N, Jeong MJ et al. Aberrant mucosal wound repair in the absence of secretory leukocyte protease inhibitor. Thromb Haemost 2004;92:288-297.

21. Ashcroft T, Simpson JM, Timbrell V. Simple method of estimating severity of pulmonary fibrosis on a numerical scale. J Clin Pathol 1988;41:467-470.

22. Livak KJ, Schmittgen TD. Analysis of relative gene expression data using real-time quantitative PCR and the 2(-Delta Delta $C(T)$ ) Method. Methods 2001;25:402-408.

23. Pezzato E, Dona M, Sartor L et al. Proteinase-3 directly activates MMP-2 and degrades gelatin and Matrigel; differential inhibition by (-) epigallocatechin-3-gallate. J Leukoc Biol 2003;74:88-94.

24. Ferry $G$, Lonchampt M, Pennel $L$ et al. Activation of MMP-9 by neutrophil elastase in an in vivo model of acute lung injury. FEBS Lett 1997;402:111-115.

25. Goodwin A, Jenkins G. Role of integrin-mediated TGFbeta activation in the pathogenesis of pulmonary fibrosis. Biochem Soc Trans 2009;37: 849-854.
26. Munger JS, Huang XZ, Kawakatsu $\mathrm{H}$ et al. The integrin alpha $\mathrm{v}$ beta 6 binds and activates latent TGF beta 1: a mechanism for regulating pulmonary inflammation and fibrosis. Cell 1999;96:319-328.

27. Hori $\mathrm{Y}$, Katoh T, Hirakata $\mathrm{M}$ et al. Anti-latent TGF-beta binding protein-1 antibody or synthetic oligopeptides inhibit extracellular matrix expression induced by stretch in cultured rat mesangial cells. Kidney Int 1998;53:1616-1625.

28. Shi M, Zhu J, Wang R et al. Latent TGF-beta structure and activation. Nature 2011;474:343-349.

29. Srivastava $M$, Steinwede $K$, Kiviranta $R$ et al. Overexpression of cathepsin $\mathrm{K}$ in mice decreases collagen deposition and lung resistance in response to bleomycin-induced pulmonary fibrosis. Respir Res 2008;9:54

30. Buhling F, Rocken C, Brasch F et al. Pivotal role of cathepsin K in lung fibrosis. Am J Pathol 2004:164:2203-2216.

31. Zhang $D$, Huang $C$, Yang $C$ et al. Antifibrotic effects of curcumin are associated with overexpression of cathepsins $\mathrm{K}$ and $\mathrm{L}$ in bleomycin treated mice and human fibroblasts. Respir Res 2011;12:154.

32. Runger TM, Adami S, Benhamou CL et al. Morphea-like skin reactions in patients treated with the cathepsin K inhibitor balicatib. J Am Acad Dermatol 2012;66:e89-e96.

33. Selman M, Ruiz V, Cabrera $S$ et al. TIMP-1, $-2,-3$, and -4 in idiopathic pulmonary fibrosis. A prevailing nondegradative lung microenvironment?s. Am J Physiol Lung Cell Mol Physiol 2000;279:L562-L574.

34. Ruiz V, Ordonez RM, Berumen J et al. Unbalanced collagenases/TIMP-1 expression and epithelial apoptosis in experimental lung fibrosis. Am J Physiol Lung Cell Mol Physiol 2003;285:L1026-L1036.

35. Selman M, Pardo A. Role of epithelial cells in idiopathic pulmonary fibrosis: from innocent targets to serial killers. Proc Am Thorac Soc 2006:3:364-372.

36. Patterson ML, Atkinson SJ, Knauper $V$ et al. Specific collagenolysis by gelatinase $\mathrm{A}, \mathrm{MMP}-2$, is determined by the hemopexin domain and not the fibronectin-like domain. Febs Lett 2001;503:158-162.

37. Clarke DL, Carruthers AM, Mustelin $\mathrm{T}$ et al. Matrix regulation of idiopathic pulmonary fibrosis: the role of enzymes. Fibrogenesis Tissue Repair 2013;6:20.

38. Oono T, Specks U, Eckes B et al. Expression of type VI collagen mRNA during wound healing. J Invest Dermatol 1993;100:329-334.

39. Specks U, Nerlich A, Colby TV et al. Increased expression of type $\mathrm{VI}$ collagen in lung fibrosis. Am J Respir Crit Care Med 1995;151: $1956-1964$ 